\title{
The Effect of Sulfurisation Temperature on Structural Properties of CuAlS 2 Thin Films
}

\author{
A. U. Moreh ${ }^{1}$, M. Momoh' 2 , B. Hamza ${ }^{3}$ \\ ${ }^{123}$ Department of Physics, Usmanu Danfodiyo University, P.M.B 2356, Sokoto, Nigeria
}

\begin{abstract}
CuAlS}_{2}$ thin films of the same thickness are deposited on suitably cleaned coning 7059 glass substrate by two stage vacuum thermal evaporation technique at room temperature and sulfurised at different temperatures. The structural properties studied by means of XRD revealed that the films are of crystalline in nature having tetragonal structure. For all the films the preferential orientation is [112]. The other orientations like [220], [312] and [400] were also observed in the films depending upon the sulfurisation temperatures. The values of lattice constants, grain size, micro strain and dislocation density of the films are calculated and the values agree strongly with ICDD data. Visual inspection of (SEM) micrographs of the films showed that crystallite size increase with increase in sulfurisation temperature.
\end{abstract}

Keywords: Dwell period, lattice parameters, Ramp rate, Sulfurisation, Thermal evaporation.

\section{INTRODUCTION}

Due to the growing interest in alternative energies, photo-voltaic has become an active field for research. Today's commercial solar cells are predominantly fabricated on the basis of crystalline silicon. However, to be used efficiently in solar cells, silicon needs to be refined to 99.999\% purity [1]. Although Silicon solar cells have produced high efficiencies, high processing costs make it unsuitable for large scale requirements. Moreover, because silicon is an indirect band-gap material, larger thickness is needed to absorb the incident sunlight. Because of these drawbacks associated with silicon, the PV industry started looking for inexpensive efficient PV cells of new semiconductors in thin film form.

The main advantage of thin film solar cells (TFSC) is that they will eventually have lower cost because:-

(i) Approximately hundred times thinner layers than $\mathrm{Si}$ are needed

(ii) They may be deposited on low cost substrates like glass, metal foils and plastic

(iii) They may be deposited by a number of inexpensive deposition techniques.

In all thin film solar cells the primary p-n heterojunctions occurs between the absorber layer and the window layer. The window layer allows the light to pass through it and travel to the absorber layer. No photocurrent is generated in the window layer. It is preferable that the semiconductor used as absorber layer be of direct band gap material.

I-III-VI ${ }_{2}$ compound semiconductors such as $\mathrm{CuAlS}_{2}, \mathrm{CuGaS}_{2}, \mathrm{CuAlSe} 2$, and $\mathrm{CuInS}_{2}$ to name a few are of interest in various device applications. Cu-chalcopyrite semiconductor materials are used as layers in heterojunction solar cells. These materials exhibit a direct band gap in the range between about 1 and $3.5 \mathrm{eV}$ and absorb the sunlight within a layer of a few microns thickness due to their high absorption coefficients of almost $10^{5} \mathrm{~cm}^{-1}$ [1] and they crystallize in the tetragonal structure. The ternary compound $\mathrm{CuAlS}_{2}$ is a promising material for its technological applications such as in opto-electronics and photovoltaic. In opto-electronics, it can be used as a light emitting diode (LED) for various electronic applications because of its wide band gap energy [2], Tariq and [3]. In photovoltaic, because of its optical and structural properties the wide gap ternary $\mathrm{CuAlS}_{2}$ crystalline thin films, could be expected as new alternative Cd-free buffer layer [4], [5]. Furthermore, these compounds could be obtained by a similar physical preparation process as the absorber layer in wide band gap photovoltaic cell WBGP [4]. CuAlS ${ }_{2}$ can also be used as a selective window coating because of its high absorption coefficient in the ultraviolet region of electromagnetic spectrum [2].

Up to now, a number of methods have been performed to produce $\mathrm{CuAlS}_{2}$ films, including, metal decomposition (MD), [6], horizontal Bridgman method, [7], Iodine transport, [8], single source thermal evaporation, [9], chemical bath deposition, (CBD) [3], [2], and spray Pyrolisis, [10]. In this work, we have succeeded for the first time in depositing $\mathrm{CuAlS}_{2}$ films on glass substrate by two stage thermal evaporation technique. The great potential of thermal evaporation such as scalability and controllability drove us to choose it as the deposition technique. 


\section{EXPERIMENTAL}

All the chemicals used (copper, aluminum and sulfur) for the deposition of $\mathrm{CuAlS}_{2}$ thin films were $\mathbf{4 N}$ grade. Corning $\mathbf{7 0 5 9}$ glass was used as substrate. Deposition of $\mathrm{Cu}-\mathrm{Al}$ alloys was performed by using EDWARDS FL 400 thermal evaporator which was equipped with SQC 310 Deposition controller. A molybdenum boat was used to evaporate $\mathrm{Cu}$ thin films and tungsten coils was used for deposition of aluminum placed at a distance of $10 \mathrm{~cm}$ from the glass substrate. The substrate temperature ranged from room temperature RT- 473K. Cu-Al precursors were converted to $\mathrm{CuAlS}_{2}$ thin films by sulfurisation/annealing using SVG 2610 BASE horizontal diffusion furnace which was equipped with mini sulfur furnace. The sulfurisation/annealing were carried out at the temperatures of $573 \mathrm{~K}, 673 \mathrm{~K}$ and $773 \mathrm{~K}$.

In order to investigate the crystallographic properties of $\mathrm{CuAlS}_{2}$ thin films, X-ray diffraction analyses were carried out using PANALYTICA XPERT PRO Diffractometer with $\mathrm{Cu}-\mathrm{Ka}$ radiation $(\lambda=1.54056 \AA)$ for the $2 \theta$ ranging from $\left(20^{\circ}-100^{\circ}\right)$. Surface morphology characterization was carried out by using EVOMA-10 scanning electron Microscope (SEM).

\subsection{Growth of $\mathrm{CuAlS} \mathrm{S}_{2}$ Thin Films}

$\mathrm{CuAlS}_{2}$ thin films were prepared by two stages:- Stage one, sequential deposition of $\mathrm{Cu}$ and $\mathrm{Al}$ layers on glass substrate to form $\mathrm{Cu}-\mathrm{Al}$ precursor, and stage two, sulfurisation of this precursor to convert it to $\mathrm{CuAlS}_{2}$. The $\mathrm{Cu}$-Al bi-layer structure was prepared on ultrasonically cleaned glass substrate by vacuum thermal evaporation of $4 \mathrm{~N}$ grade copper and aluminum in a sequential mode. Molybdenum boat was used as source for the deposition of copper and tungsten coils was used for deposition of aluminum. In these way samples of $\mathrm{Cu}-\mathrm{Al}$ precursors of $100 \mathrm{~nm}$ thickness were grown at room temperature (RT).

Sulfurisation deposited is the conversion of metallic $\mathrm{Cu}-\mathrm{Al}$ thin films to $\mathrm{CuAlS}_{2}$ thin films by reaction in sulfur vapor at a given temperature. In this work conversion process of the $\mathrm{Cu}-\mathrm{Al}$ thin films to $\mathrm{CuAlS}_{2}$ was carried out by Sulfurising $\mathrm{Cu}-\mathrm{Al}$ thin films in an elemental sulfur vapor in 3 steps at the temperatures of 573K, $673 \mathrm{~K}$ and $773 \mathrm{~K}$ at a ramp rate of $10^{\circ} / \mathrm{min}$, the dwell period was set to one hour and sulfur vapor was allowed to diffuse into the sample at the rate of $4.3 \mathrm{Sccm}$ by using Argon as a carrier gas.

\section{RESULTS AND DISCUSSION}

Fig. 1a shows XRD patterns for as deposited samples, it is clear that no meaningful diffraction peaks were observed, this implies that the films show no evidence of molecular crystallization, the grain size of the film was so small suggesting that the disorder within these grains is so high leading to amorphous nature. Thus in this case films grown at a low substrate temperature such as room temperature and did not undergo post thermal treatments are usually randomly oriented and amorphous. Similar observation was reported by [11] on thermally evaporated $\mathrm{Cu}$-In precursors and has accounted that the formation of amorphous $\mathrm{Cu}$-In thin films by evaporation when deposition was carried out at a temperature between room temperature and $426 \mathrm{~K}$ was attributable to incomplete reaction at such low temperatures.

Fig. 1b, 1c, and $1 \mathrm{~d}$ depict samples sulfurised at $573 \mathrm{~K}, 673 \mathrm{~K}$ and $773 \mathrm{~K}$ respectively. It is observed that films sulfurised at $573 \mathrm{~K}$ (fig $1 \mathrm{~b}$ ) were characterized by two main crystalline peaks; the first peak appeared at $2 \theta$ $\sim 28^{\circ}$ while the second peak appeared at $2 \theta \sim 48^{\circ}$. On comparing with ICDD (card no. 01-074-7042) and reported values of [4] and [12], the first peak observed at $2 \theta \sim 28^{\circ}$ was identified to be belonging to $\mathrm{CuAlS}_{2}$ chalcopyrite structure with the (112) preferred orientation, the second peak observed at $2 \theta \sim 48^{0}$ is designated to (220) orientation also belongs to $\mathrm{CuAlS}_{2}$ chalcopyrite structure.

Fig. 1c depicts XRD patterns for samples sulfurised at 673K. It is observed that in addition to first and second peaks exhibited by thin films grown at room temperature, a third peak appeared at $2 \theta \sim 55^{\circ}$ and is designated to (312) orientation and belongs to crystalline $\mathrm{CuAlS}_{2}$ chalcopyrite structure as confirmed by ICDD (card no. 01-074-7053) and previous work of [7] and [13].

Fig. 1d illustrates the diffraction patterns for samples sulfurised at 773K. As seen from the figure fourth peak emerged and is assigned to (400) reflections of $\mathrm{CuAlS}_{2}$ in accordance with ICDD (card no. 01-075-6862). The emergence of reflection peaks with increase in sulfurisation temperature is a clear improvement in crystallinity of the films. [14] attributed this to interdiffusion of sulfur and restructuring of particles at a sulfurisation temperature itself during sulfurisation process. For films grown at very low substrate temperature, sulfurisation temperature augment particle's gain in thermal energy which enhances their restructuring, enlarge grain size and reduce defects thus leading to crystalline film. 


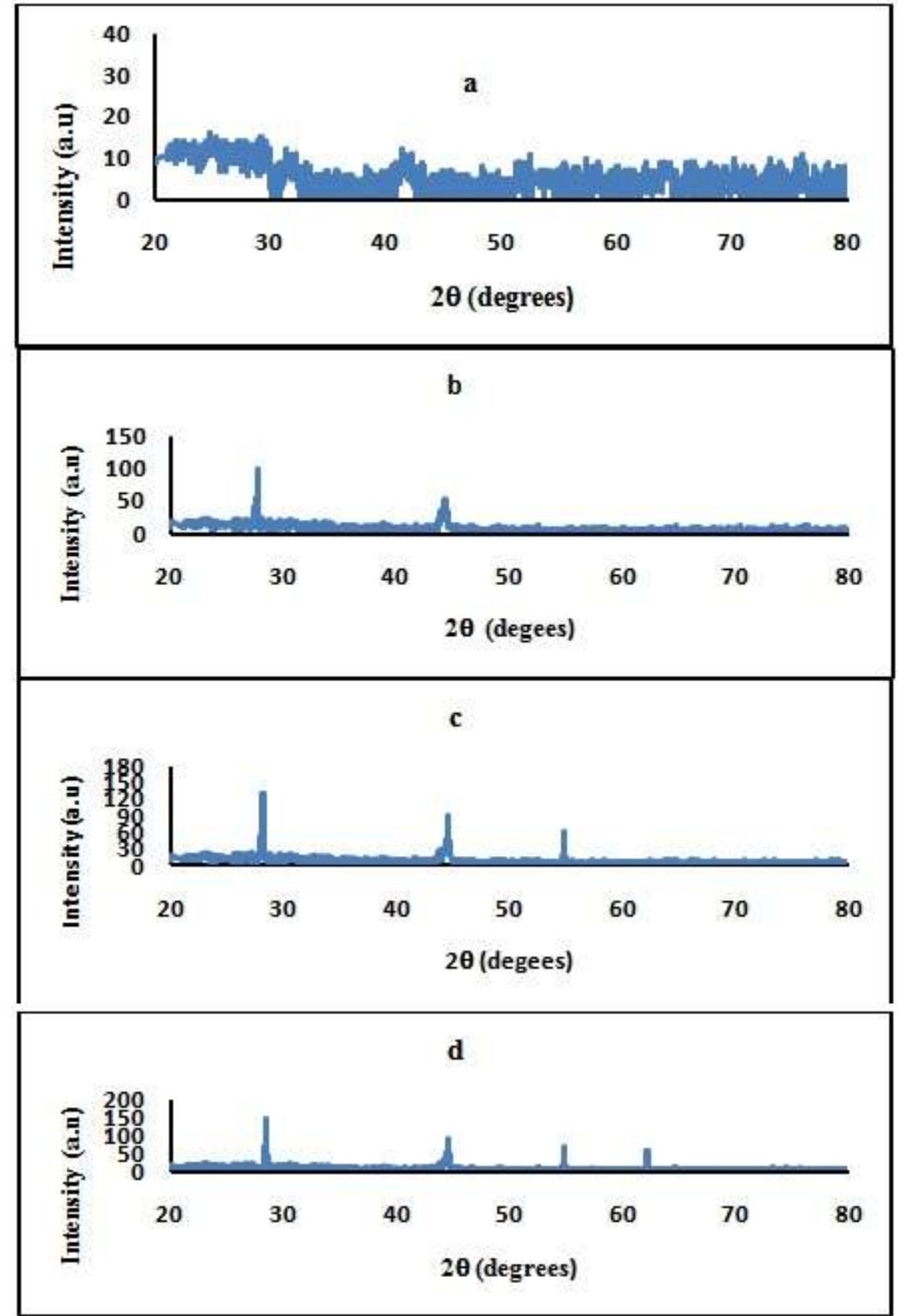

Figure 1 XRD patterns for (a) as deposited (b) sulfurised at 573K (c) sulfurised at 673K (d) Sulfurised at $773 \mathrm{~K}$

The grain sizes were calculated through the Scherer's formula

$\mathrm{D}=\frac{0.9 \lambda}{\beta \cos \theta}$

Where $\mathbf{D}$ is the grain size, $\beta$ experimentally observed diffraction peak width at Full wave half maximum intensity (FWHM), and $\theta$ is Bragg angle. The Dislocation density of thin films were calculated by employing the relation [10]

$\delta=\frac{n}{D^{2}}$ 
The micro strain $(\varepsilon)$ developed in thin films was calculated from the relation [15].

$\varepsilon=\frac{\beta \cos \theta}{4}$

Where $\beta=$ FWHM (Full width at half maximum intensity.

The lattice parameters a and c value for tetragonal crystallographic system can be calculated from the following equation using $h k l$ parameter and the inter planer spacing $\mathbf{d}$

$\frac{1}{d^{2}}=\frac{h^{2}+k^{2}}{a^{2}}+\frac{l^{2}}{c^{2}}$

Tables 1.0, display the lattice parameters for samples grown at room temperature, and sulfurised at $573 \mathrm{~K}, 673 \mathrm{~K}$ and $773 \mathrm{~K}$. It is observed from the table and as demonstrated in fig. 2 that for all the films the grain size $\mathbf{D}$ increases with increases sulfurisation temperature. However the dislocation density $\boldsymbol{\delta}$, micro strain $\boldsymbol{\varepsilon}$ and FWHM decreases with increase in sulfurisation temperature this is depicted in fig. 3, 4 and 6 respectively. At the highest sulfurisation temperature i.e. $773 \mathrm{~K}$, the grain size increases to as high as $145 \mathrm{~nm}$, whereas $\boldsymbol{\delta}$, $\boldsymbol{\varepsilon}$ and FWHM decreases to as low $0.47 \times 10^{14}$ lines $/ \mathrm{m}^{2}, 2.39 \times 10^{-3}$ and 0.0010 respectively. These increase in grain size and decrease in $\boldsymbol{\delta}, \boldsymbol{\varepsilon}$ and $\mathbf{F W H M}$ showed that at $773 \mathrm{~K}$ thin films are crystalline and much less strained.. Our calculated values of $\boldsymbol{\delta}$ and $\boldsymbol{\varepsilon}$ are close to those reported by [7] and Nishi [6]. The lattice constants a and $\mathbf{c}$ agree well with the standard values.

Table 1.0 Structural Parameters of $\mathrm{CuAlS}_{2}$ thin films

\begin{tabular}{|c|c|c|c|c|c|c|c|c|c|}
\hline \multirow{3}{*}{$\begin{array}{c}\text { Sample } \\
\text { (nm) }\end{array}$} & \multirow{3}{*}{$\begin{array}{l}\text { Sulfurisation } \\
\text { Temp. (K) }\end{array}$} & \multicolumn{4}{|c|}{ Lattice constants } & \multirow[t]{3}{*}{$\mathrm{D}(\mathrm{nm})$} & \multirow{3}{*}{$\begin{array}{c}\delta \times 10^{14} \\
\text { lines } / \mathrm{m}^{2}\end{array}$} & \multirow{3}{*}{$\begin{array}{c}\varepsilon \\
\times 10^{-3}\end{array}$} & \multirow[t]{3}{*}{ FWHM } \\
\hline & & & & c & & & & & \\
\hline & & Calc. & ICDD & Calc. & ICDD & & & & \\
\hline 100 & 573 & 5.51 & \multirow{3}{*}{5.33} & 11.12 & \multirow{3}{*}{10.44} & 75 & 1.78 & 4.62 & 0.0020 \\
\hline 100 & 673 & 5.47 & & 10.46 & & 105 & 0.90 & 3.30 & 0.0015 \\
\hline 100 & 773 & 5.35 & & 10.45 & & 145 & 0.47 & 2.39 & 0.0010 \\
\hline
\end{tabular}

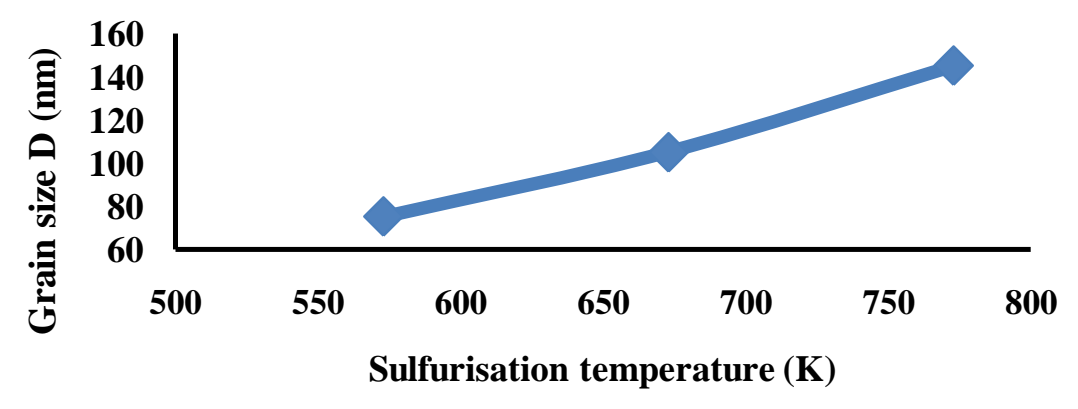

Figure 2.0 grain size as a function of sulfurisation temperature

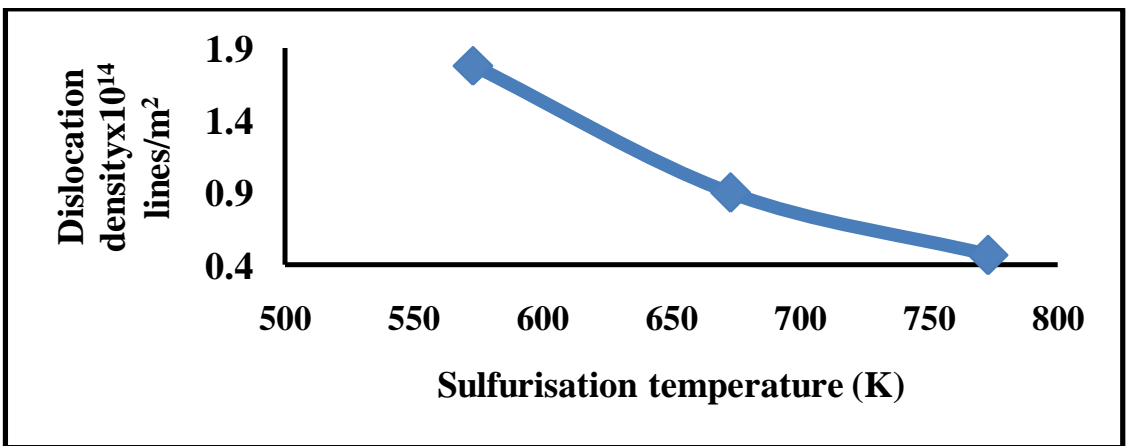

Figure 3.0 dislocation density as a function of sulfurisation temperature 


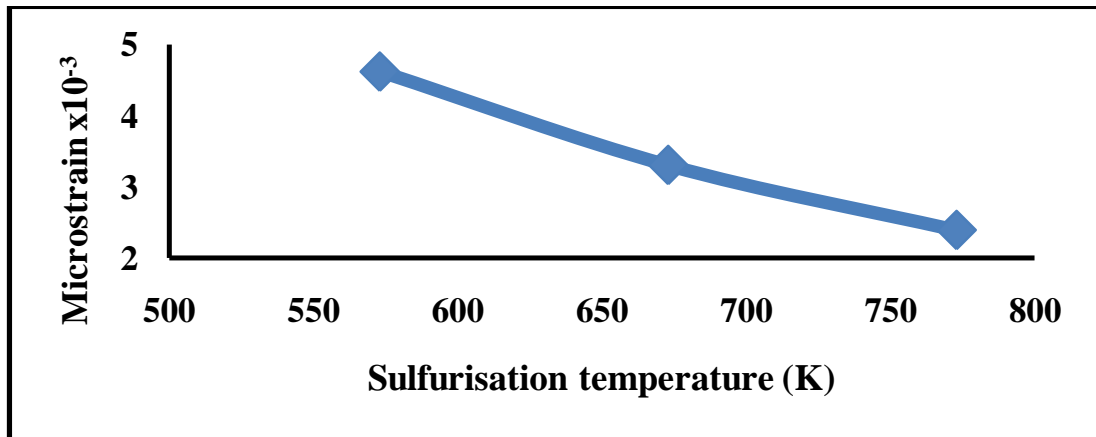

Figure 4.0 micro strains as a function of sulfurisation temperature

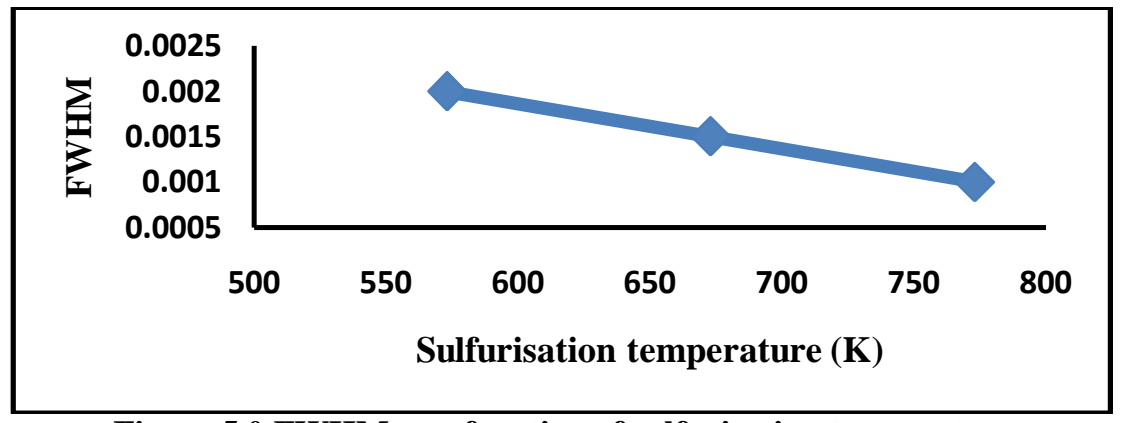

Figure 5.0 FWHM as a function of sulfurisation temperature

The FWHM $\beta$, decreases appreciably with increasing temperature of sulfurisation. The decrease of FWHM indicates the improvement in the crystallinity of the films during sulfurisation.

Fig. 6a-c depicts films grown at RT but sulfurised at 573K, 673K and 773K respectively. Fig. 6a indicates the micrographs of CuAlS2 thin films deposited at RT and sulfurised at 573K. It is observed that a cluster of tiny spheres are irregularly distributed over the surface of the film. Similarly lot of empty space is observed within these clusters. The cluster size shows remarkable rise with sulfurisation temperature which confirms improvement in grain size [16]. The random distribution of grains and size suggests a random nucleation mechanism and random orientation of grains show that the grain growth is isotropic [17]. Fig. 6b shows that as the sulfurisation temperature reaches $673 \mathrm{~K}$ a large number of spheres appeared some of which appeared to be larger than those formed in fig. 6a. This, we believe is an indication of overgrowth of the particles which imply an increase in grain sizes of the films with increase sulfurisation temperature. In the same vein a careful look at fig. $6 \mathrm{c}$ shows that a bigger but fewer crystallites are formed as sulfurisation temperature rose to $773 \mathrm{~K}$. The variations observed in grain sizes on sulfurisation at different temperatures could be attributed to the structural modifications witnessed in XRD analysis. When the grain sizes obtained from both XRD and SEM measurements are compared, there is a large difference, the reason being the grain sizes appeared in SEM images are the grains only at the surface of films, while grain size calculated from the XRD is the average value of the grain sizes distributed throughout the thickness of the film. Similar observation was made by [18].

(a)

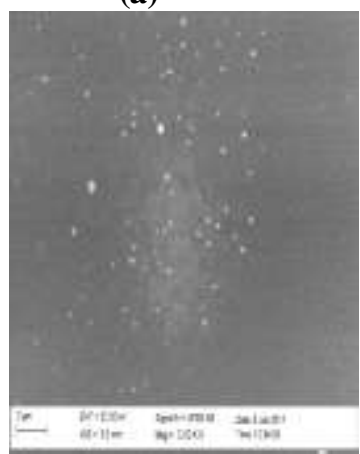

Figure 6.0 SEM Micrographs for films deposited at RT and sulfurised at (c)
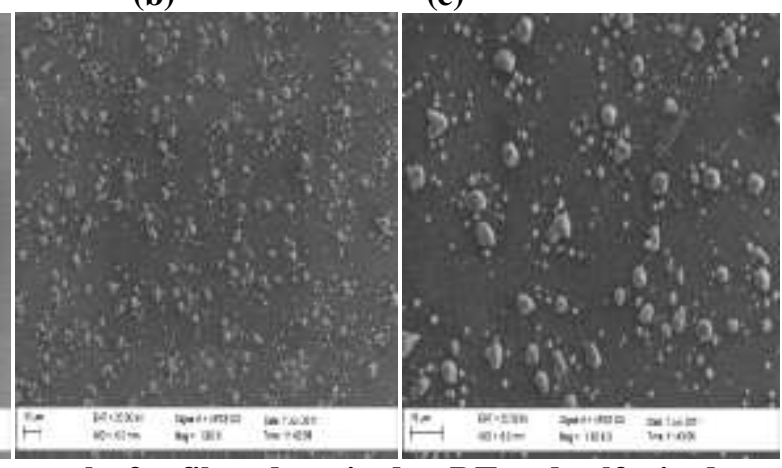

(a) $573 \mathrm{~K}$ (b) $673 \mathrm{~K}$ (c) $773 \mathrm{~K}$ 


\section{CONCLUSION}

The deposition of $\mathrm{CuAlS}_{2}$ film was successfully carried out on a glass substrate (glass slide) at room temperature using two stage vacuum thermal evaporation techniques. XRD characterizations reveal that sulfurisation temperature has a great influence on the structure of films. It was discovered that the crystallinity of the grown films increase with increasing sulfurisation temperature. Similarly lattice parameters were found to depend largely on sulfurisation temperature. The grain size $\mathbf{D}$ of the thin film increases with increasing sulfurisation temperature, while micro strain $(\varepsilon)$, dislocation density $(\delta)$, and FWHM $(\beta)$ were found to decrease appreciably with increasing sulfurisation temperature and this renders the samples less strained and crystalline especially at highest sulfurisation temperature $(773 \mathrm{~K})$. This work would benefit the fabrication and investigation of optoelectronic and photovoltaic devices.

\section{ACKNOWLEDGEMENT}

We acknowledge the Advanced Physics Lab SHESTCO, Abuja for providing research facilities for carrying out the work. We are also thankful to TETFUND for financial support.

\section{REFERENCES}

[1] O. Madelung, Semiconductors Data Handbook. Springer- Verlag Berlin, Heidelberg, 2004

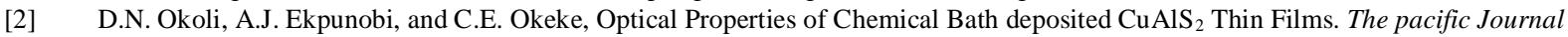
of Science and Technology, 2006,7, 59-63.

[3] J.A. Tariq, J.A and A.J. Mushtak, Structure and optical properties of CuAlS2 Thin films by CBD, Turk. Journal of Physics, 34, 2010, 107- 116 .

[4] C.O. El Moctara, K. Kambas, S. Marsillac, U.A. Anagnostopoulos, J.C. Bern`ede, and K. Benchouck, Optical Properties of $\mathrm{CuAlX} 2(\mathrm{X}=\mathrm{Se}, \mathrm{Te})$ thin Films obtained by Annealing of Copper, Aluminum and Chalcogen layers sequentially deposited. Thin Solid Films, 2000, 371.

[5] P.B. Ahirrao, S.R. Gosavi, S.S. Sanjay and R. S. Patil, Wide band gap Nanocrystalline CuSCN thin films deposited by Modified Chemical Method. Archives of Physics Research. 2, 2011,: 29-33.

[6] T. Nishi, Preparation of $\mathrm{CuAlS}_{2}$ by Metal Organic Decomposition, Japanese Journal of Physics, 38, 1999, 626-628.

[7] R. Brini, G. Schmer, M. Kanzari, and B. Rezig, Study of Growth of CuAlS 2 thin Films on Oriented Silicon (111), Journal of Solid Thin Films, 517, 2009, 2191-2194.

[8] M. Hidetone, Y. Michitaka, and S. Koichi, Vapor phase Epitaxy of CuAlS 2 on $\mathrm{CuGaS}_{2}$ Substrate by the Iodine transport Method. Journal of Crystal Growth, 153, 1995, 180-183.

[9] M. Abaab, A. S. Bouazzi, and B. Rezig, Competitive CuAlS 2 Oxygen Gas sensor. Microelectronic Engineering, 51, 2000, 343-348

[10] I. Illican, Y. Caglar, and M. Caglar, Structural, Morphological and ptical Properties of CuAlS $\mathrm{C}_{2}$ thin films deposited by Spray Pyrolisis, Journal of Optoelectronics and Advanced Materials, 10, 2008, 10.

[11] A. Aldrin, Preparation and characterization of certain II-VI, I-III- VI2 Semiconductor thin films and transparent conducting oxides. PhD Thesis, Cochin University of Science and Technology India, 2004,

[12] S. Agilan, D. Mangalaraj, S.K. Narayandass and G. Mohan Rao, (Unpublished), Effect of Thickness and Substrate Temperature on Structure and Optical Band gap of Hot wall-deposited CuInSe2 Polycrystalline Thin Films.

[13] C. J. Olejni ceka, L.E. Flannerya, S.A. Darveau, C.L. Exstroma, S. Kment, N.J. Iannob, N.J. and R.J. Soukup, CuIn ${ }_{1-x} A_{1} l_{x} 2$ thin Films Prepared by Sulfurization of Metallic Precursors. Journal of Alloys and Compounds, 509, 2011, 10020-10024.

[14] A. Bouloura, K. Djessas, and D. Todorovic (2009). Structural and Optical Properties of Cu (In,Ga)Se2 Grown by Closed-spaced Vapor transport Technique. Material Science in Semiconductor Processing, 7, 2009, 82-87.

[15] K. Sarma, R. Sarma, and H.L. Das, Structural Characterization of Thermally Evaporated CdSe thin Films. Chalcogenide Letters, 5, 2008, 153-163.

[16] A. U. Ubale, V.P. Deshpande, and D. P. Gulwade, Electrical, Optical and Structural Properties of Nano- structured Sb2S3 Thin Films deposited by CBD Technique, Chalcogenide Letters. 7,2010, 101-109.

[17] R. Al-Gaashani, S. Radiman, N. Tabet and A. R. Daud (2011). Synthesis and optical Properties of CuO Nanostructures obtained via a novel Thermal decomposition Method. Journal of Alloys and Compounds, 35, 2011, 8761-8769.

[18] Matsuo, H., Yoshino, K. and T. Ikari (2006). Thin Solid Films. 515: 505. 